Notas

\title{
Campesinado, agricultura familiar y un Trabajo Social agroecológico
}

Sergio Díaz Angarita

Licenciado en Trabajador Social

(Universidad Minuto de Dios)

Correo: orrnak12@gmail.com 


\section{Resumen}

Este artículo exhibe los resultados del trabajo investigativo titulado "Agricultura Familiar, Campesinado y su incidencia en la construcción del Trabajo Social Agroecológico", el cual demuestra la importancia de la agricultura familiar en el modo de vida del campesinado y sus aportes hacia un Trabajo Social agroecológico, es decir, un Trabajo Social enfocado en el estudio del campo, la ruralidad, la conservación del medio ambiente, sus paisajes, las transformaciones de sus poblaciones, sus prácticas de vida y su cultura.

Para ese trabajo se toma como metodología la hermenéutica; como método de investigación el cualitativo y su enfoque la etnografía; para la recolección de la información se usaron: la historia de vida, la observación participante y la revisión documental. Los resultados evidencian que para el pequeño campesino no industrializado la agricultura familiar es la mejor alternativa para resistir al sistema económico capitalista dominante, dentro de la producción de bienes y servicios agrícolas, demostrando que ésta es una de las mejores prácticas para conservar sus conocimientos ancestrales y compartirlos con otros interesados en el tema. Por otro lado, el Trabajo Social puede enriquecer la agricultura familiar en la medida que da continuidad a la visión de la agroecología desde sus prácticas y esas alternativas de vida que tiene el campesinado en relación con el labriego, el cuidado de la tierra, el medio ambiente y la conservación de todo hábitat, sin olvidar la construcción de relaciones sociales entre los campesinos que se suman a estas nuevas prácticas en la agricultura.

Palabras claves

Trabajo Social - Agricultura familiar - Agroecología 


\section{Abstract}

This article shows the results of the research project titled 'Family Farming, Peasantry and its impact on the construction of Agroecological Social Work'. This project demonstrates the importance of Family Farming as a way of life for the small farmer, contributing to the construction of an Agro-ecological Social Work, in other words, a Social Work focused on the study of the rural sector, rurality, conservation of the environment, the landscapes, and the transformations of the peasant populations close to the central cities, consolidating the conservation of their practices and the ancestral culture. For this work, we used a hermeneutical, qualitative method and its focus was ethnography. In order to collect information, life stories, participant observation and written documents were used. Results demonstrate that Family Farming is part of the effective alternative strategies to resist the dominant economic system. This alternative not only focuses on the production of agricultural goods and services, but also on the importance of establishing relationships of trust, demonstrating that the conservation of ancestral knowledge has the possibility of resisting and of being shared with those who wish to learn about it. Furthermore, Social Work could complement Family Farming by perpetuating the Agro-Ecologic framework within its practices and the alternative lifestyles that rural populations have to offer in relation to taking care of the Earth and the environment, as well as conserving any type of habitat, without forgetting about the importance of social relations between farmers, which are included within these new agricultural practices.

\section{Keywords}

Social Work - family farm - Agro-Ecology 


\section{Introducción}

La agricultura familiar en Colombia, como en muchas partes del mundo, se enmarca en la consolidación cultural de grupos minoritarios que trabajan arduamente en la construcción de soberanía alimentaria dentro de las naciones, y que son representadas por "campesinos, indígenas, afrodescendientes, agricultores urbanos y neo-rurales que viven en condición de exclusión y abandono" (Acevedo, Osorio \& Martínez Collazos, 2016). La agricultura familiar es un proceso de fortalecimiento a los grupos individuales como a los colectivos desde sus conocimientos, formas de producción, apropiación de territorios y de entender los procesos de mejoramiento en temas rurales (desarrollo rural, ordenamiento territorial, uso de tecnologías, etc.). Asimismo, forma parte de los procesos alternativos que luchan contra un sistema capitalista industrial que amenaza su extinción definitiva. De igual forma, los agricultores familiares como sus familias hacen parte de esta elección, ya que es una alternativa que busca la reivindicación de derechos, la consolidación de su autonomía, y la resistencia sociopolítica en conjunto.

La estructura social agrícola en Colombia está distribuida en pequeños, medianos y grandes productores agrícolas, destinados a la producción de café, flores, banano, caña de azúcar, palma aceitera, arroz, algodón, maíz, soya, sorgo, papa, frutas, hortalizas y cultivos de economía campesina (cebolla larga, habas, cebolla cabezona, papa, frijol, cacao, arveja, zanahoria), como también en la parte pecuaria de ganado, carne, leche, avicultura y porcicultura (Fenwarth, 2017).

El Tercer Censo Nacional Agropecuario —CNA— de 2014 arrojó importantes datos sobre la realidad rural colombiana. Por ejemplo, mostró que el $82 \%$ de los cultivos agrícolas representan el 6,3\% del total del área rural dispersa, lo que equivale a 7 millones $115 \mathrm{mil}$ hectáreas. De los 2,7 millones de productores censados, se identificaron 524 mil jefes de hogar, de los cuales 122 mil son mujeres, es decir el 23,2\%. Igualmente, de los productores residentes censados, el 36,6\% son mujeres (Acevedo, Osorio \& Martínez Collazos, 2016:3). 
Siguiendo esta perspectiva, el cierre del año 2015 y el balance preliminar para el 2016, se puede observar que la producción del sector agrícola tuvo sólo un $1.1 \%$ de crecimiento. Con excepción de arroz (12.9\%), palma de aceite $(9.9 \%)$, cacao $(15.8 \%)$ y frutales $(7.1)$, que mostraron importantes incrementos en sus niveles de producción; los demás cultivos tuvieron comportamientos moderados e incluso descensos significativos, de acuerdo con la información gremial recopilada por la Sociedad de Agricultores de Colombia (SAC). En este caso, cultivos relacionados con la economía campesina del pequeño y mediano productor, cultivos como maíz $(-18.4 \%)$, sorgo $(-36.7 \%)$, cebada $(-41,3 \%)$, trigo $(-42,9 \%)$, algodón $(-22,8 \%)$, tabaco $(-13,1 \%)$, soya $(-8.8 \%)$, frijol $(-8.2 \%)$ y hortalizas $(-4.4 \%)$. El comportamiento negativo de la producción de estos cultivos, en primera medida es por su ciclo corto en su mayoría, está asociado a la reducción de áreas sembradas, y por condiciones climáticas desfavorables provocadas por el fenómeno de El Niño (López, 2016).

Entendiendo lo anterior, el panorama es complejo. Para los pequeños y medianos productores, su existencia en estas condiciones es casi nula, dado que ellos hacen parte de las políticas del gobierno de turno y de las iniciativas de "revolución verde" a nivel mundial, lo cual los excluye al no entrar en el mercado y el cultivo extensivo de soya, caña de azúcar, palma aceitera, ya que su producción es principalmente para el autoconsumo, y lo restante es para la comercialización, y el desarrollo de iniciativas de comercio justo, en mercados locales, y consumo solidario, para lo cual deben sustentarse en la calidad de sus productos para alcanzar ganancias significativas y equitativas.

Este artículo se relaciona, en particular, con una investigación realizada en el área de Trabajo Social a un campesino de la vereda La Pradera del municipio de Subachoque, en Cundinamarca, Colombia. La Pradera es una vereda ubicada en el municipio de Subachoque a unos 56 kilómetros de Bogotá, por la vía calle 80, la cual dividió al pueblo de Madrid. Para llegar allí se debe recorrer Puente de Guaduas, Siberia, Puente de Piedra, La Cuesta, el Rosal y Subachoque, donde está el destino, a unos 10 kilómetros del pueblo (Díaz, 2015:20). 
Cabe resaltar que el pueblo es miembro activo de la Asociación Red Agroecológica Campesina (ARAC).

El tema central de la investigación es resaltar la importancia de la agricultura familiar en la producción alimentaria y cómo ésta forma parte de múltiples iniciativas de conservación y cuidado de la cultura campesina desde sus tradiciones de labriego de la tierra, de la producción de economía campesina, demostrando que esta iniciativa se convierte en una opción de vida para el pequeño y mediano campesino que desde la tenencia de su tierra pertenece a un territorio y su identidad a una territorialidad.

El sistema capitalista actual para la parte rural data de iniciativas sustentadas en políticas públicas favorables para la industria de los bio-combustibles, la producción a gran escala de cultivos favorables a la idea de "reducción de la pobreza" — como lo especifica la revolución verde (maíz, soja, palma aceitera) - y con el crecimiento del sector pecuario. En temas relacionados a la expansión y utilización masiva de las tierras, para la producción de carne y leche, cabe aclarar que los que pueden desarrollar estas iniciativas son los campesinos industrializados, ganaderos, ya que cuentan con el capital suficiente - a nivel económico, y en extensión masiva de tierraspara implementar estas estrategias del mercado, desplazando así las alternativas, es decir, a la agricultura familiar. Para este caso, relataré cómo pequeños campesinos organizados y no industrializados han encontrado una alternativa para la producción, comercialización y mantenimiento de un ecosistema saludable que dé vida, para la producción alimentaria, la preservación cultural y el poder enseñar las formas de hacer, mantener relaciones sociales por solidaridad, esto es, relaciones de ayuda mutua, conocimientos compartidos y expresiones de reconocerse como una familia extendida en la comprensión y la manutención de su quehacer matutino, las cuales no se ven tan afectadas por dicho sistema, ya que se conocen entre sí y comparten espacios de crecimiento, unidad y colectividad organizada.

Ante esto, la mejor opción parece ser la agricultura familiar. No obstante, son pocos los estudios que se conocen respecto a este tema, razón por la cual el interés de la investigación en la que se basa este 
artículo es mostrar la importancia de la agricultura familiar para las zonas rurales cercanas a la ciudad y las que están distantes de la misma, compartiendo y hablando desde los pequeños campesinos no industrializados y sus aportes a la construcción de un Trabajo Social enfocado al estudio del campo, su ruralidad, y que éste pueda demostrar el apoyo a los procesos organizativos de las comunidades y de las familias que hacen parte de esas acciones e iniciativas desde un concepto como lo es la agroecología. Desde el Trabajo Social se puede evidenciar esa nueva tipología de familia, "familia por solidaridad", nacida desde los procesos organizativos y desde la autonomía que se da a partir del reconocimiento propio y la gestión de conservación territorial y la resistencia por su soberanía, autonomía y autosuficiencia alimentaria.

La investigación parte de comprender el concepto de agricultura familiar desde las prácticas culturales y desde la producción alimentaria de la ARAC, contraponiéndolo al concepto de agricultura familiar postulado por la Asamblea General de las Naciones Unidas para la Alimentación y la Agricultura (FAO, en sus siglas en inglés), y sus aportes al ejercicio profesional del Trabajo Social.

La agricultura familiar comprende algunos conceptos clave, tales como: agroecología, campesinado, organización social, territorio y territorialidad; conceptos necesarios para comprender y ampliar la perspectiva que se posee sobre la agricultura familiar, dado que ésta va más allá de un conjunto de actividades sociales, económicas y culturales, de hombres, mujeres y jóvenes de zonas rurales que practican tradicionalmente la labranza de la tierra y se relacionan desde el trabajo con otros sujetos/actores sociales que tienen las mismas prácticas.

En ese sentido, en primer lugar, se define la agroecología como aquella ciencia que propende al estudio y manejo ecológico de los recursos naturales, teniendo en cuenta la compatibilidad y retroalimentación entre los saberes tradicionales y la investigación científica. Asimismo, se plantea como un enfoque distinto de la agricultura convencional-tradicional hegemónica que acelera procesos naturales con la utilización de agroquímicos, ligado al medio ambiente y a lo 
social, centrado no sólo en la producción sino en la sostenibilidad ecológica dentro del sistema productivo y diversificado dentro de su propio terreno. Así pues, la agroecología se debe entender como la relación existente entre el trabajo del agricultor y la producción de la tierra, también como una apuesta por el cuidado del medio ambiente y el fortalecimiento sociocultural de las comunidades rurales y periurbanas, que son partícipes de tradiciones, saberes y costumbres. Bajo esta perspectiva, la agroecología hace parte fundamental de la agricultura familiar, en tanto que reconoce, por una parte, el sentido cultural y de identidad que los campesinos le dan a la tierra y, por otra parte, el trabajo que ellos desarrollan con ésta, teniendo en cuenta sus prácticas ancestrales que han sido un legado de preservación de generación en generación.

En segundo lugar, aunque son variados los conceptos acerca del campesinado, hay algo en lo que coinciden las distintas perspectivas y es que el campesino es aquel sujeto que tiene una relación directa con la tierra; que cosecha para sí mismo, su familia y su comunidad. Es un sujeto/actor social que como ser histórico muestra a los demás las contradicciones y sus tendencias al desarrollo. Cumpliendo un rol social y cultural a medida que comparte sus conocimientos y tradiciones, enseñando a los demás esos modos de interpretación a la vida y a la toma de decisiones que afectan a su ser como al de sus semejantes con el medio ambiente y logrando interactuar de igual manera con los nuevos aprendizajes.

En tercer lugar, como lo indica Correa (2010), se entiende por organización social el producto histórico de las relaciones de mutuo acuerdo entre sujetos sociales de una comunidad que se organizan para su producción y reproducción social; abarcando aspectos económicos, ideológicos, culturales (Díaz, 2015), lo cual incluye transformaciones de sí mismos como resultado de sus propias dinámicas históricas.

Finalmente, el territorio hace referencia a la ocupación de un espacio terrestre; en él se ve una relación de poder y/o de posesión por parte de uno o varios individuos. Sin embargo, no existe límite a esta definición, pues también se puede entender como un lugar de "terri- 
torialidad" que va más allá de la ocupación de un espacio, abarcando también la apropiación cultural y simbólica del mismo, como en el caso de los campesinos, para quienes sus tierras representan no sólo un símbolo de trabajo sino también un símbolo de vida, memoria, identidad, resistencia, de alternativas y de adaptaciones al cambio.

En ese orden de ideas, se entiende la agricultura familiar como un modo de vida para el campesinado, el cual comienza desde su producción alimentaria, su relación con sus semejantes y con el compartir de su cultura. Desde esta premisa es necesario reconocer que tanto para ellos como para instituciones gubernamentales (FAO) y no gubernamentales (ARAC) este ejercicio se convierte en un trabajo de resistencia, lucha y alternativa sociopolítica para entender el progreso de las áreas rurales de un país.

Para el año 2014 la asamblea general de las Naciones Unidas y su oficina para la Alimentación y la Agricultura (FAO), declaró el Año Internacional de la agricultura familiar. Su objetivo en dicha conmemoración fue visibilizar y fortalecer la agricultura familiar, entendida como agricultura a pequeña escala que tiene como finalidad erradicar la pobreza y el hambre, comprendiendo y conduciendo al desarrollo sostenible desde el sector rural para implementar la seguridad alimentaria. A partir de la declaración del Año de la Agricultura Familiar, la FAO consideró la necesidad de definir en qué consistía aquella iniciativa: "una forma de organizar la agricultura (ganadería, silvicultura, pesca, acuicultura y pastoreo), que es administrada y operada por una familia y, sobre todo, que depende preponderantemente del trabajo familiar, tanto de mujeres como hombres. La familia y la granja están vinculados, co-evolucionan y combinan funciones económicas, ambientales, sociales y culturales" (2014:26).

Siguiendo la línea de la FAO, el campesinado tiene una función vital en la agricultura familiar, pues en sus manos se encuentra la forma de producción alimentaria. No obstante, aunque la FAO busque mejorar las condiciones de vida en las zonas rurales, sus postulados al centrarse en el desarrollo sostenible y la tecnificación del campo ocasionan que se dejen de lado los métodos tradicionales de labranza 
de la tierra y, junto con esto, que se pierdan las prácticas ancestrales y la cultura de sus tradiciones.

La FAO muestra que para tener una agricultura familiar consolidada o al menos en transición, se debe acceder a créditos, tener grandes extensiones de tierra y acceder a la mercantilización de la tierra, ahí se piensa en el campesino industrializado que debe emplear nuevos métodos de labrar la tierra y poseer el uso de las nuevas tecnologías, nuevas semillas certificadas (genéticamente modificadas), y destinar sus esfuerzos a la mercantilización de la producción (biocombustibles, palma aceitera, caña de azúcar) desde la apertura a mercados externos para sus productos, es decir que deben pensar más en la exportación que en el mercado local. Razón por la cual, los pequeños y medianos campesinos no son tenidos en cuenta y son considerados "pobres": porque no se centran en la mercantilización de la tierra y el uso de semillas certificadas-transgénicas, sino que se concentran en la producción de alimentos sanos, de calidad, cotidianos (frutas, hortalizas) y sustentan su mercado local, compartiéndolos con la comunidad, en solidaridad, vecindad, familiaridad, y en el enseñar sus costumbres y creencias ancestrales a aquellos que se acercan y los reconocen como actores importantes en el sostenimiento de la seguridad alimentaria.

Debido a esa concepción de agricultura familiar de la FAO, podemos entender sus intenciones de forma mercantilista en el uso de la tierra (carácter con el que no están de acuerdo muchos de los campesinos), y reconocer que desde organizaciones no gubernamentales como el Grupo Semillas, ARAC, Cultivadores de vida SUC (Suma paz Usme, Ciudad Bolívar), Federación Nacional Sindical Unitaria Agropecuaria (FENSUAGRO-CUT), Federación Nacional de Cooperativas Agropecuarias (FENACOA), Movimento dos Trabalhadores sem Terra (MST), Movimento dos Pequenos Agricultores (MPA), Vía Campesina, entre otros, se está trabajando para que la agricultura familiar sea entendida desde otra perspectiva y sea reconocida por la mayoría de las población (rural y urbana y reivindicada desde la labranza de la tierra a través de espacios no institucionalizados, visibilizando prácticas ancestrales, recuperando 
la memoria colectiva, conservando el medio ambiente, y afianzando la pedagogía desde el pensamiento agroecológico, que se sustenta en el fortalecimiento de la organización colectiva, oponiéndose a la idea de la revolución verde, a la siembra de semillas transgénicas y de cultivo extensivo (que tiene varias consecuencias negativas sobre la tierra, como infertilidad, incendios forestales, desviación de ríos, no preservación de bosques nativos, erosión masiva).

Para la ARAC, la agricultura familiar es la actividad económica principal del campesino, donde la familia y el agricultor son el centro de la organización empresarial. La tierra, desde la perspectiva del campesinado, no solamente es un producto activo, sino que forma parte de su identidad y de su patrimonio material y cultural. La agricultura familiar del pequeño y mediano campesino, como lo señala don Pedro Gonzales (campesino de La Pradera y miembro activo de la ARAC), consiste en mantener sus rasgos, su tierra, su vegetación, su todo; mientras que, para el campesino agroindustrial, el interés está en la economía y el hecho de estar fortalecidos económicamente (Díaz, 2015). Así pues, la agricultura familiar para el pequeño y mediano campesino, resulta ser una alternativa y opción de vida para resistir a las políticas excluyentes del sistema industrial dominante que quiere la ruralidad de las naciones. La ARAC, a diferencia de la FAO, más allá del beneficio propio, busca beneficiar a toda a una comunidad, es decir, a su familia, enseñando que cada uno puede tener un espacio para hacer labriego de la tierra y de ella alimentarse, como también proporcionando alimentos sanos, fortaleciendo sus lazos sociales, y mostrando otra alternativa de vida.

\section{Metodología}

La propuesta metodológica de esta investigación deriva de un cúmulo de distintos saberes, y pretende mostrar que se puede enfocar el Trabajo Social al campo de la agroecología; ya que es necesario sentar posición y poseer visión crítica para comprender las estrategias 
económicas y políticas que hoy afectan a muchas regiones del mundo en su ruralidad.

Lo anterior se realiza, en un primer momento, partiendo del análisis del concepto de agricultura familiar desde la ARAC para contraponerlo al concepto planteado por la FAO. En un segundo momento, realizando un trabajo de campo en el que se acompaña a un campesino en sus labores diarias, con el propósito de conocer la manera en que labra la tierra y mantiene una relación de familiaridad, respeto y honra, conociendo su cultura y la concepción que tiene acerca de la agricultura familiar y sus derivados (agroecología, organización social, territorio, educación, política). En ese orden de ideas, la investigación parte de la hermenéutica como enfoque metodológico para interpretar los discursos dados por otros y comprarlos; en la praxis se centra la etnografía como el método utilizado para llevar a cabo el trabajo de campo; la metodología empleada fue la cualitativa. Como métodos de recolección de la información se usaron: entrevista semiestructurada, historia de vida, observación participante, revisión documental y análisis crítico del discurso.

\section{Resultados}

Tras realizar el trabajo etnográfico y la revisión documental, uno de los resultados que se obtuvo fue que, dentro de las comunidades campesinas no industrializadas, se identifican roles específicos que se transforman con el tiempo, en donde el trabajo de la agricultura familiar no es puesto solamente en manos de las mujeres, sino que el hombre, desde sus tradiciones, fomenta el fortalecimiento del trabajo del labriego de la tierra, reconociéndose como un actor fundamental para la apropiación del territorio y la territorialidad desde su identidad y la consolidación de espacios asertivos donde muchos actores pueden unir sus fuerzas para crear espacios de resistencia.

De igual modo, se evidenció que la agroecología posibilita escenarios participativos para la construcción de la soberanía, autonomía, seguridad y autosuficiencia alimentaria; rescatando y preservando los 
saberes ancestrales del campesinado, resaltando la cultura campesina y la resistencia que ésta hace desde una economía propia en contra de la lógica del sistema capitalista, la cual consiste en la acumulación de capital y exportación a nuevos mercados.

Asimismo, se obtuvo que los aportes que se pueden hacer desde el Trabajo Social a la agricultura familiar se relacionan con dar continuidad a la visión de la agroecología y con mostrar que existen alternativas de vida dentro del sistema dominante, porque lo importante es dar el campo al campesino que en verdad cree en él, que sabe de su cuidado, del trabajo con la tierra y de la preservación del medio ambiente.

La agricultura familiar busca articular múltiples iniciativas para crear y enseñar desde sus postulados que es posible llegar al desarrollo de una reforma integral para el sector rural de las naciones, donde sus mercados locales se conviertan en iniciativas productivas de seguridad, soberanía alimentaria, protegiendo los recursos ambientales (agua, biodiversidad, agro diversidad), reconociendo saberes ancestrales y el buen uso de las tecnologías agrícolas (sistematización de la agricultura, sistemas de riego, drones y robots, agricultura protegida, acceso y uso de las telecomunicaciones), demostrando que se puede y debe tener políticas diferenciales e integrales de reconocimiento de los actores y sus derechos.

El Trabajo Social como profesión debe conocer y entender cómo acciones concretas buscan desde las relaciones económicas, políticas y culturales de forma horizontal la extensión de ideas cotidianas dentro del territorio nacional, desde las organizaciones sociales, el fomento y fortalecimiento del tejido social para que exista la continuidad cultural como una alternativa a los procesos de globalización, demostrando la cercanía y la construcción de nuevos vínculos para resistir desde la solidaridad y la innovación comunitaria. 


\section{Conclusiones y discusión}

Tomando en cuenta los resultados obtenidos, en primer lugar, es importante decir que al Trabajo Social aún le falta investigar mucho más en las zonas rurales. No obstante, en la investigación aquí señalada se destaca la idea de que el Trabajo Social se enfoque en un trabajo agroecológico, posibilitando el acercamiento de éste a los procesos comunitarios para fomentar estrategias que exalten la vida cultural de las mismas comunidades y para evidenciar dentro de ellas transformaciones que se resisten a los procesos económicos del sistema dominante.

Así, el Trabajo Social agroecológico "consiste en conocer, trabajar, recopilar, formular e iniciar una propuesta ética, política y metodológica en la construcción y fortalecimiento de las organizaciones sociales que demuestran la importancia de su trabajo desde el sector rural, exaltando su cultura" (Díaz, 2015:78-79). De este modo, importa entender los discursos hegemónicos que se enseñan sobre la realidad rural de cada país, reconocer el aporte y la comprensión de nuevas propuestas dentro del medio ambiente, el desarrollo territorial, un hábitat para todos y por qué no una propuesta donde todos hagamos un mundo más agradable para vivir.

En segundo lugar, es importante entender que el sistema capitalista está configurando al campesino para hacerlo entrar en las dinámicas del modelo de privatización del campo, de mercantilización de sus servicios; a lo que el campesinado se opone retomando sus formas tradicionales de labrar la tierra, rescatando su cultura e identidad desde sus historias, sus semillas y sus organizaciones de base. El campesinado se define "por la unificación de proyectos comunes que tienden hacia una síntesis de su subjetividad, tomando como partida unas condiciones sociales compartidas: la tierra, tal producción le permite, a su vez, el sostenimiento familiar y en ocasiones un excedente, el cual lo articula casi siempre con mercados locales y regionales y la construcción de entornos comunales de reconocimiento" (Díaz, 2015:40). 
Finalmente, ante las dinámicas planteadas por el sistema económico y ante la perspectiva de la FAO de la agricultura familiar, el campesinado resiste creando su propia concepción de agricultura familiar, la cual le proporciona autosuficiencia y seguridad alimentaria, teniendo en cuenta principalmente sus prácticas ancestrales y dejando de lado los postulados internacionales que buscan una privatización del campo modificando toda una tradición campesina, aplicando nuevas técnicas en la forma de labranza, la utilización de nuevas semillas y la apertura a mercados externos para la mercantilización de sus productos. Invisibilizando, a la vez, al campesino como actor fundamental para el sostenimiento alimentario de una sociedad urbana, que sólo entiende dinámicas de consumo y no de preservación y de mercados conscientes destruyendo la relación del comerciante con el productor, disponiendo nuevos vínculos de tejido consciente de consumo productor-consumidor.

\section{Referencias bibliográficas}

ACEVEDO, OSORIO, A., y MARTínEZ COLLAZOS, J. La Agricultura Familiar en Colombia: estudios de casos desde la multifuncionalidad y sus aportes a la paz. Bogotá, Colombia, Fondo Editorial Ediciones Universidad Cooperativa de Colombia, Coorporación Universitaria Minuto de Dios, Agrosolidaria, 2016.

CORREA RUBIO, F. Elementos de identidad y organización social entre las comunidades indígenas de la región del vaupés. Manguaré (2), 2010.

DÍAZ, A. Agricultura Familiar, Campesinado y su incidencia en la construcción del Trabajo Social Agroecológico. Bogotá, Colombia: Corporación Universitaria Minuto de Dios.

FENWARTH, E. A. (07 de 02 de 2017). Portafolio, 2015. [en línea: 11/07/2017] Disponible en: http://www.portafolio.co/opinion/andres-espinosa-fenwarth/ situacion-actual-y-perspectivas-agropecuarias-2017-503226

LÓPEZ, R. M. (26 de 01 de 2016). SAC. [En línea: 12/07/2017]. Disponible en: http:// www.sac.org.co/es/estudios-economicos/balance-sector-agropecuario-colombiano/290balance-y-perspectivas-del-sector-agropecuario-2012-2013.html

SALCEDO, S. Y GUZMÁN, L. (Ed). Agricultura familiar en América Latina y el Caribe: recomendaciones políticas. Santiago, Chile, FAO, 2014. 
Sergio Díaz Angarita

SILVA, D. F. Asociaciones Campesinas en resistencia Civil Construcción de Paz y Desarrollo del Magdalena Medio. Bogotá, Colombia, Corporación Universitaria Minuto de Dios, 2011. 